\title{
La práxis del contrabajo en los Conservatorios Elementales y Profesionales en España
}

\section{The praxis of double bass in Elementary and Professional Conservatories in Spain}

\author{
Paula Hernández-Dionis \\ Conservatorio Superior de Música de Canarias \\ trolonchelo@gmail.com \\ ORCID iD: https://orcid.org/0000-0002-6735-9822
}

\section{RESUMEN}

El presente estudio trata de conocer la metodología empleada en torno a este instrumento en los Conservatorios Elementales y Profesionales del país. La recogida de información se ha realizado a través de un cuestionario en el que han participado 47 profesores de 43 conservatorios de todas las comunidades autónomas salvo Melilla, comunidad en la que no existe profesor de esta especialidad. El resultado nos muestra la heterogeneidad de la metodología utilizada por el profesorado del instrumento, quien adapta la programación a sus circunstancias locales y al alumnado que tiene. Asimismo, destaca la inquietud del profesorado por dar más visibilidad al instrumento más grande de la familia de la cuerda, pues en multitud de casos no disponen del material necesario para impartir estas enseñanzas.

Palabras clave: contrabajo, conservatorio profesional, conservatorio elemental, metodología, praxis.

\section{Abstract}

The present study aims to assess the methodology used around this instrument in the Elementary and Professional Conservatories of the country. The information was collected through a questionnaire in which 47 teachers from 43 conservatories from all the 
autonomous communities participated except for Melilla, a community in which there is no teacher for this speciality. The results show the heterogeneity of the methodology used by the teachers, who adapt the programming to their local circumstances and the students they have. Likewise, it highlights the concern of the teachers to give more visibility to the largest instrument of the string family, since in many cases they do not have the necessary material to impart this discipline.

Key Words: double bass, professional conservatory, elementary conservatory, methodology, praxis.

Hernández Dionis, P. (2021). La práxis del contrabajo en los Conservatorios Elementales y Profesionales en España. Cuadernos de Investigación Musical, (13), pp. 92-105.

\section{INTRODUCCIÓN}

La transformación del contrabajo a lo largo de los siglos, ha estado condicionada por varios factores. Las diferentes formas y utilidades que se le dio en los distintos países, así como la diferencia en su tamaño y afinación son algunos de ellos (Ballesteros, 2004). Entre los siglos XV y XVIII existió una gran diversidad de instrumentos, muchos de ellos iguales, pero denominados de forma diferente (frecuentemente, como violone). Eran construidos de manera distinta dependiendo de la nacionalidad del luthier. Estos instrumentos eran afinados en terceras, cuartas, quintas o una mezcla de estos intervalos y con distinta cantidad de cuerdas (entre tres y seis).

Las primeras referencias a un instrumento predecesor al contrabajo lo encontramos en autores como Próspero, Francolini, o Amman, quienes en el siglo XV y XVI ya hablaban en sus libros y relieves sobre instrumentos graves de gran tamaño. Según Almenara (2007), existen diferentes líneas de investigación sobre el origen del contrabajo según los diferentes investigadores: 
PAUla HeRnández DiONiS

\begin{tabular}{|c|c|}
\hline Investigador & Línea de investigación \\
\hline Alfred Planyavsky & 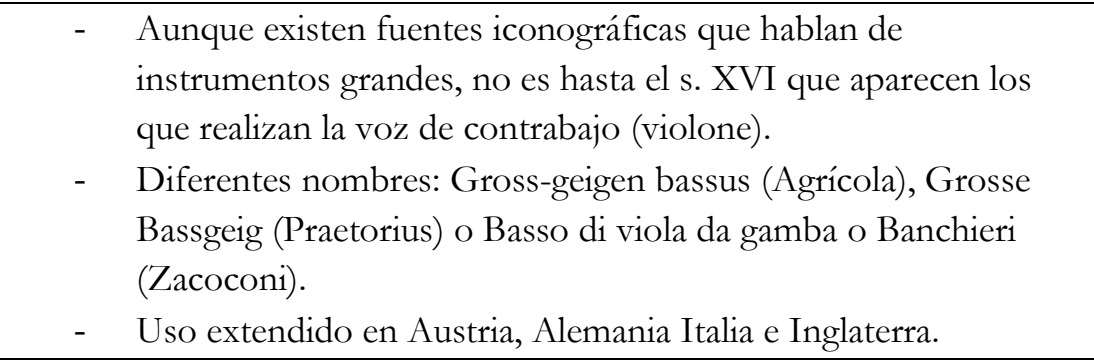 \\
\hline $\begin{array}{l}\text { Joëlle Franchen } \\
\text { Morton }\end{array}$ & $\begin{array}{l}\text { - } \quad \text { A finales del s. XV, se importan de España e Italia } 4 \text { tipos } \\
\text { diferentes de violas graves: dos bajos de viola y dos violones. } \\
\text { - } \quad \text { De menor difusión, el Morton Violone in contrabasso. }\end{array}$ \\
\hline $\begin{array}{l}\text { Stephen Bonta y } \\
\text { Therald Borgir }\end{array}$ & $\begin{array}{l}\text { - Bonta defiende que el violone no se usaba para tocar notas en } \\
\text { una tesitura subgrave, sino siempre en la grave. } \\
\text { - Borgir defiende que el término violone se utilizó en el siglo } \\
\text { XVII. }\end{array}$ \\
\hline $\begin{array}{l}\text { Marc } \\
\text { Vanscheeuwijck }\end{array}$ & $\begin{array}{ll}\text { - } & \text { Estudia el violone en Italia en el s. XVII. } \\
\text { - } & \text { El término violone se refiere a una viola da braccio. } \\
\text { - } & \text { En tesitura grave, se refieren a una viola da gamba (grande, } \\
\text { grosso, doppio, contrabasso, in contrabasso, etc.) }\end{array}$ \\
\hline Paul Brun & $\begin{array}{l}\text { - Toma como punto de partida los instrumentos de la familia del } \\
\text { violín. } \\
\text { - Documento francés de } 1656 \text { habla de "basse de viollon façon de } \\
\text { Lorrain". } \\
\text { - Praetorius habla de "Gran Bajo". } \\
\text { - } \quad \text { En } 1662 \text { aparece "la grosse basse". }\end{array}$ \\
\hline Michael Greeberg & $\begin{array}{l}\text { - Estudia el periodo que comprende desde el principio del s. XVI } \\
\text { hasta } 1815 . \\
\text { - Es imposible determinar la fecha de la introducción del } \\
\text { contrabajo en Francia. }\end{array}$ \\
\hline $\begin{array}{l}\text { Xosé Crisanto } \\
\text { Gándara }\end{array}$ & $\begin{array}{ll}\text { - } & \text { Estudia la aparición del contrabajo en España. } \\
\text { - } & \text { La familia de las violas, es una evolución de las vihuelas de arco } \\
& \text { renacentistas del s.XV. } \\
\text { - } & \text { El término violone en España se utilizaba "para todos los } \\
& \text { instrumentos de la familia del violín" (Almenara, 2007, p.29). }\end{array}$ \\
\hline
\end{tabular}

Tabla 1: Líneas de investigación sobre el origen del contrabajo según Almenara (2007) (Elaboración propia).

Es con el desarrollo de la música de cámara y de otros instrumentos, como los de viento metal y percusión, que durante el s.XVIII el contrabajo toma un mayor protagonismo en países como Italia. No será hasta el final de esa centuria, que aumente en más de 150 obras la literatura de este instrumento con función solista. (Hernández-Dionis, 2017). Ya en el s. XIX, comienza a aparecer el término de contrabbasso de la mano del investigador vienés Planyavsky, quien hace referencia a la tesitura del instrumento (Crisanto, 1996). Durante el s.XX, las composiciones para contrabajo comenzaron a florecer tanto en su versión solista, 
como de música de cámara u orquesta. Además, se generaliza el uso de cuerdas entorchadas y de clavijas metálicas y se le da más importancia a su papel como instrumento solista.

La enseñanza del contrabajo en este siglo (Almenara, 2007), se desarrolla de la mano de grandes maestros en los distintos países. En Italia, Isaia Billé y Francesco Petracchi destacan en la primera y segunda mitad del siglo respectivamente. Ludwig Streicher, en Austria, fue la voz más influyente del contrabajo a mitad del s. XX, desarrollando una amplia labor como solista. Destacó además por la publicación compuesta por cinco volúmenes de su método My way of playing the double bass (Streicher, 1997) en el que desarrolla ampliamente, entre otras cosas, la técnica de arco alemán (consistente en coger el arco, según la escuela, a la manera de la viola da gamba).

Por último, Edouard Nanny y Jean-Marc Rollez en Francia, desarrollan distintos métodos para contrabajo, destacando por la técnica de arco francés (a diferencia del arco alemán, con el arco francés, el instrumentista coge el arco por encima de la vara) el primero y por la técnica de mano izquierda el segundo, quien desarrolla ejercicios de escalas y ejercicios con el fin de mejorar la técnica y afinación del instrumentista. Rollez, fue además el inventor de un arco a caballo entre el alemán y el francés, cuyo talón era más grande y tenía más espacio, no tanto como en el alemán, pero si más que en el francés. De esta manera se podía ejercer una mayor presión y se lograba con ello que las crines no rozaran la madera a la hora de tocar (Hernández-Dionis, 2017).

En España, comienza la educación musical reglada con el Real Conservatorio de Música de María Cristina, cuya fundación data del 15 de julio de 1830. Fue creado por el Rey Fernando VII como obsequio a su esposa, amante de la música. Tuvo como primer director a D. Francisco Piermarini y entre su alumnado (de ambos sexos) coexistían los internos (de 12 a 15 años) y los externos (hasta los 18). En la Real Orden donde se establece este mandato, se habla ya de un profesor de contrabajo, estableciendo en el plan de estudios diario, un programa horario para este instrumento de 8 a 11 horas todos los días. En 1838, se modifica el plan de estudios por medio de una Real Orden en la que se especifica que el alumnado de contrabajo, debería tener como mínimo 12 años. El 20 de junio de 1868, el Real Decreto firmado por el ministro de fomento D. Severo Catalina, establece un cambio en la denominación del Real Conservatorio, pasando a llamarse Escuela de Música y Declamación. Poco después, en diciembre del mismo año, se declara disuelto el Conservatorio de Música y Declamación y se crea la Escuela Nacional de Música, estableciendo de nuevo un profesor de contrabajo que cobraría 1500 pesetas.

La Reina regente, en nombre de Alfonso XIII, decretó en 1901 el cambio de nomenclatura de la Escuela Nacional de Música y Declamación, a la de Conservatorio Nacional de Música y Declamación. El 16 de junio de 1905, se establece el Real Decreto por el que se regula la creación de nuevos conservatorios y en 1917, el Reglamento para el gobierno y el régimen del Real Conservatorio de Música y Declamación. Es en el Boletín del Estado del 4 de julio de 1942, cuando se establece por primera vez que los conservatorios deberían ser divididos en Superiores, Profesionales y Elementales, estando el contrabajo, en el caso del Real Conservatorio de Madrid, dentro del apartado de clases. 


\section{Paula Hernández Dionis}

Posteriormente, las distintas leyes existentes (Decreto 2618 /1966, Reales Decreto 1073/1987, 1104/1990 y 970/1994, Ley Orgánica 1/1990, Reales Decretos 756/1992, 1463/2001, 2194/2004, Ley Orgánica 2/2006) y las leyes de organización académica de las distintas Comunidades Autónomas han sido las bases reguladoras de las enseñanzas del contrabajo en los conservatorios elementales y profesionales españoles.

El profesorado que ha ejercido en estos conservatorios ha sido muy extenso desde que se fundaron, pero haremos a continuación una pequeña reseña de aquellos que han aportado una mayor transcendencia en la didáctica del contrabajo en España. Según Crisanto (2000), en el Real Conservatorio de Madrid o Escuela Nacional de Música (según el momento), destacaron nombres como José Venancio López, Manuel Muñoz, Juan José Torres o Emilio M. Lluna. En Cataluña, se funda en 1837 el Conservatorio del Liceo con profesores de clara ascendencia italiana, como Ramón Mainés, Eduard Oliveras o Antón Torelló. En el Conservatorio Municipal de Música barcelonés, fundado en 1886, destacaron nombres como José Bassas, profesor de Ferran Sala o Francisco Lozano. Pedro Valls Durán (1869-1935), alumno de Giovanni Bottesini, fue profesor de contrabajo en la Escuela Municipal de Música de Barcelona y uno de los mejores pedagogos del s. XIX. (Crisanto, 2000)

El desarrollo del arco alemán en España fue aumentando cuando Ludwig Streicher llegó al país, en un principio impartiendo clase en diversos cursos por todo el territorio nacional y luego instaurando la cátedra en la Escuela Superior de Música Reina Sofía. Son numerosos los profesores de los conservatorios españoles que fueron alumnos de él y continúan con sus enseñanzas hoy en día. Otros profesores que destacaron junto con Streicher en la enseñanza del contrabajo en España fueron Ferrán Sala (Barcelona), Javier Sapiña (Valencia y Aragón), Lucián Cioratta (procedente de Rumanía, fue profesor en Sevilla) y Karen Martirossian (nacido en Azerbaiyán, fue profesor en Madrid) (Ximo Clemente y Zlatka Pencheva, Comunicación personal, 1 de marzo de 2021).

Actualmente, en las Enseñanzas Superiores de Música, podemos señalar a profesores tan importantes en el panorama internacional como Antonio García Araque (Escuela Superior de Música Reina Sofía), Julio Pastor (Musikene), Ximo Clemente (Aragón), Jorge Ángel Muñoz (Málaga) o Jonathan Camps y Uxía Martínez (Escuela Superior de Música de Cataluña). En los Conservatorios Profesionales de Música, podemos destacar a profesores como Hugo Valero (Murcia), Diego Tejedor (Zaragoza), Xavier Puertas (Girona) o Virginia de Vega (Madrid).

\section{OBjetivos}

El objetivo principal de esta investigación es conocer la metodología empleada por los profesores de contrabajo en los conservatorios españoles, tanto profesionales como elementales. Este objetivo general nos lleva a plantearnos los siguientes objetivos específicos:

- Mostrar el perfil del profesorado actual de contrabajo.

- Conocer alumnos de contrabajo existentes en estos conservatorios a nivel nacional. 
- Ahondar acerca de las ratios utilizadas en las clases individuales de contrabajo y su eficacia en estas enseñanzas.

- Dar a conocer los elementos técnicos más utilizadas por el profesorado de la asignatura con los distintos tipos de alumnos.

- Revelar los grupos existentes en los distintos conservatorios sobre este instrumento.

- Exponer la bibliografía y métodos más estudiados por el alumnado a nivel nacional.

- Estudiar la importancia dada a la posición corporal, así como al ejercicio y a los estiramientos necesarios para tocar este instrumento.

- Analizar la facilidad existente para organizar cursos y masterclass de contrabajo en los distintos conservatorios.

- Determinar si el material existente en las aulas de contrabajo es suficiente para impartir la docencia.

\section{Metodología}

La metodología utilizada para esta investigación, se basa en un estudio mixto, tanto cualitativo como cuantitativo. Como baza principal, contamos con el cuestionario realizado vía online entre los meses de febrero y junio del año 2020, al profesorado de contrabajo de los conservatorios españoles. En este cuestionario, en el que se recoge la información con una metodología mixta, el profesorado manifiesta su opinión sobre la metodología empleada en su aula. Además, se han seguido las siguientes pautas para desarrollar esta investigación:

- Revisión de la historia de los conservatorios en España.

- Breve revisión bibliográfica en fuentes primarias y secundarias sobre lo escrito hasta el momento sobre el instrumento.

- Repaso generalizado del desarrollo de las enseñanzas del contrabajo en España en el último siglo.

\subsection{FUENTES DE INFORMACIÓN}

Para la realización del presente estudio de investigación se han utilizado las siguientes fuentes de información:

- Profesores participantes en el cuestionario realizado.

- Programaciones didácticas de los conservatorios españoles.

- Bibliografía consultada, así como el estudio en fuentes primarias de la legislación relativa a la historia de los conservatorios españoles. 


\section{PAula Hernández Dionis}

\subsection{PROCEDIMIENTO DE RECOGIDA DE LA INFORMACIÓN}

Como se ha señalado anteriormente, el cuestionario realizado ha sido la principal fuente elegida para registrar la información. Según Bisquerra (1989), un cuestionario es uno de los métodos más utilizados para recoger información, siempre por escrito, de los sujetos de una investigación.

Nuestro cuestionario posee dos tipos de preguntas: abiertas y cerradas. Además, posee una serie de preguntas de elección múltiple, que por medio de una escala permiten ofrecer varias alternativas al que responde (Rodríguez, Gil \& García, 1996). Asimismo, los propósitos con los que ha sido diseñado este cuestionario, responden a los objetivos planteados en esta investigación.

El cuestionario realizado está formado por 6 secciones de contenido diferenciado. La primera sección corresponde a la caracterización de la muestra, con preguntas como el nombre completo, edad, sexo, lugar de nacimiento, centro de trabajo, lugar de estudios o nombre de los principales profesores.

En segundo lugar, se recoge la información sobre las características de la docencia impartida, figurando entre las preguntas realizadas, la cantidad de alumnos en cada grado a los que se enseña, asignaturas impartidas, años de docencia, tipo de técnica utilizada y enseñada, ratios y grupos existentes.

Seguidamente, se indaga en el tipo de repertorio, obras, métodos y partituras utilizadas en cada centro con los alumnos de contrabajo. El cuarto apartado, dirigido a la técnica del contrabajo, reúne datos sobre los elementos técnicos que se imparten en las clases y el tiempo dedicado a este menester en cada una de ellas. La importancia del cuerpo con respecto al instrumento, ocupa la quinta posición en las secciones de este cuestionario. Se realizan preguntas como la importancia dada a esta cuestión, los ejercicios realizados para fortalecer la musculatura, etc.

La última sección del cuestionario está dedicada a la organización y capacidad tanto económica como material de los centros. La organización de cursos y masterclasses, además de los instrumentos y banquetas con los que se cuenta y sus características son parte de las preguntas de este apartado.

\subsection{POBLACIÓN Y MUESTRA}

La población de esta investigación la constituyen todos los profesores de contrabajo de los conservatorios españoles. Aunque se desconoce con exactitud el número de conservatorios existentes, sabemos que son aproximadamente 250, entre conservatorios y centros autorizados. En muchos de ellos no hay profesor de contrabajo, como en el conservatorio de Melilla, única Comunidad Autónoma sin representación en esta investigación. 
La muestra la componen 47 profesores de 43 conservatorios distintos de todas las Comunidades Autónomas, salvo la anteriormente mencionada, y con una amplia representación de provincias del país.

\section{RESUltados}

Los datos obtenidos en el cuestionario realizado, nos presentan la realidad sobre la praxis del contrabajo en los Conservatorios Elementales y Profesionales de España.

En primer lugar, podemos observar las características de la muestra de la población observada. De las 47 personas que respondieron al cuestionario, 11 de ellas (23\%4\%) son mujeres y $36\left(76^{\prime} 5 \%\right)$ son hombres.

Sobre su edad, observamos que el profesor más joven de los que ha respondido, tiene 27 y el mayor 62. De ellos, 9 se encuentran en el rango de edad de entre 27 y 39 años, 21 entre 40 y 49, 15 entre 50 y 59 y tan sólo 2, entre 60 y 62. De estos datos podemos considerar que la mayoría del profesorado de contrabajo de los conservatorios españoles tiene entre 40 y 49 años.

Sobre el lugar de procedencia del profesorado, podemos destacar las provincias y comunidades españolas de Salamanca, Madrid, Barcelona y Valencia. Asimismo, observamos que también hay profesorado procedente de otros países como Cuba, Argentina, Bulgaria o Francia.

El perfil de los docentes a nivel profesional es muy dispar, aunque podemos sacar los siguientes resultados de la muestra consultada:

En el conjunto de docentes, 2 profesores llevan entre 10 y 19 años tocando el contrabajo, 17 entre 20 y 29 años, 24 de ellos entre 30 y 39 años y tan solo 4 de ellos entre 40 y 47 años.

Como principales profesores de los docentes actuales, destacan los nombres de Javier Sapiña (7), Antonio García Araque (6), Franco Petracci (4), Ludwig Streicher (3) y Ángel Santafe (2).

El profesorado ha estudiado en países como Estados Unidos, Austria, Cuba, Francia, Holanda, Inglaterra o Suiza. En cuanto a los centros musicales destacan: CSM de Castellón, CSM de Córdoba, CSM de Sevilla, CSM de Canarias y Escuela Superior de Música Reina Sofía.

De entre los docentes consultados, 27 de ellos compaginan su actividad laboral en los conservatorios tocando con distintos grupos de cámara o música tradicional, 16 tocando en diferentes orquestas del país, ya sea de forma ocasional o perteneciendo a alguna de ellas y 4 de ellos no trabaja ni toca en otros lugares.

Entre otras actividades musicales a destacar, observamos las siguientes: especialistas en música de jazz (4), publicación de sus propios métodos didácticos (3), grabación de discos 


\section{Paula Hernández Dionis}

tanto como solistas como con distintas orquestas (3) y técnico de sonido, especialista en instrumentos de plectro y especialista en música antigua (1).

La mayoría toca otros instrumentos como puede ser el piano (16), bajo eléctrico (13) o la guitarra (11).

Algunos de ellos también son licenciados y especializados en otras materias no musicales como: Geografía e Historia, Psicología y Fotografía.

Un $53^{\prime} 1 \%$ de los encuestados utiliza la técnica de arco alemán, un 25’5\% la técnica de arco francés y un $21^{\prime} 2 \%$, ambas.

Cuando tocan como solista, un $42 \% 5 \%$ de la muestra toca de pie, un 34\% toca sentado y un $23 \%$ toca de ambas maneras, dependiendo de la situación y repertorio a interpretar.

Entrando de lleno en la praxis del contrabajo en los conservatorios, encontramos que 8 de los encuestados llevan menos de 10 años impartiendo docencia en los mismos, 19 entre 10 y 19 años, 17 entre 20 y 29 y sólo 3 de ellos llevan más de 30 años. Es por ello por lo que podemos deducir, que es en los últimos 25 años, donde se ha aumentado el número de alumnos de contrabajo en los centros y por tanto la cantidad de profesores para impartir la docencia.

Debido a la baja cantidad de alumnos matriculados, se le completa al profesorado su jornada laboral con otras asignaturas como Música de Cámara (22), Orquesta (7) o Lenguaje Musical (3). Tan sólo el 234\% de la muestra, manifiesta que tiene uno o más compañeros profesores de contrabajo trabajando en el mismo centro.

El número de alumnos matriculados en este instrumento, como venimos reflejando anteriormente, es escaso. Incluso, observamos que seis de los profesores encuestados declaran que solo tienen alumnos en las Enseñanzas Elementales (en adelante EE), siendo la media de alumnos por profesor es de 6'04 alumnos. Sin embargo, en las Enseñanzas Profesionales (en adelante EP), la media de alumnos desciende hasta los 5’74.

En algunos conservatorios se utiliza actualmente la pedagogía de ratio 3, consistente en impartir clase de instrumento a tres alumnos a la vez favoreciendo el intercambio de conocimientos e impresiones entre el alumnado. En otros, la ratio es de 2 alumnos en $1^{\circ} \mathrm{y}$ $2^{\circ}$ de EE. Sin embargo, la mayoría, el $87^{\prime} 2 \%$ de ellos declaran impartir clase de contrabajo en ratio uno frente al 12'7\%, que declaran hacerlo en ratio de dos o más alumnos. En EP, este porcentaje disminuye considerablemente al $2^{\prime} 1 \%$ del profesorado que imparte docencia en ratio múltiple frente a un mayoritario $85^{\prime} 1 \%$ que imparte en ratio uno.

Entre las razones expuestas destacan la incomodidad del espacio disponible, al ser instrumentos muy grandes, la dedicación exclusiva al alumno en el caso de la clase individual a nivel técnico, artístico y psicológico, los diferentes niveles técnicos y necesidades de cada alumno y la falta de material didáctico de calidad.

La mayoría de los encuestados coinciden en que, con la clase grupal o colectiva de contrabajos, se consigue trabajar aspectos técnicos del instrumento, la motivación, sociabilización y la consciencia grupal. 
Sin embargo, no en todos los conservatorios existe este tipo de agrupaciones ya que tan solo las encontramos en un $38^{\prime} 2 \%$ de los centros. Entre ellos destacan la Contraband del Conservatori de Cervera (8 alumnos), Grupo de contrabajos del Conservatori de Barcelona, Grupo de Contrabajos del CPM de Alcazar de San Juan o El Ship Sound Ensemble (5 alumnos) del CPM de Cartagena.

En cuanto a los aspectos técnicos del instrumento, encontramos la predominancia de la enseñanza del arco alemán (44'6\%) frente a la enseñanza del arco francés (23'4\%) en los conservatorios. Un $31^{\prime} 9 \%$ declara enseñar ambas técnicas de arco a sus alumnos.

Por otro lado, observamos que un $42 \% 5 \%$ preparan a sus alumnos para tocar tanto sentado como de pie, un $38^{\prime} 2 \%$ solo de pie y un $19^{\prime} 1 \%$ enseñan a tocar sentado.

Entre los métodos o partituras más utilizadas para impartir docencia en EE, podemos destacar entre otras múltiples opciones los siguientes: All for strings: String bass. de Gerald E. Anderson y Robert S. Frost (17), Suzuki bass School de Shinichi Suzuki (13), New method for the double bass de F. Simandl (12) o Contrabajo para niños de Hugo Valero (4).

El 808\% de la muestra, da la máxima importancia a la enseñanza de la técnica en la docencia del contrabajo. Es por ello que, aunque en las EP predomina la utilización de obras específicas para el instrumento, se utilizan muchos métodos de estudio de escalas o técnica propia del instrumento. Entre estos sobresalen: New method for the double bass y Complete Etudes de F. Simandl (28), My way of playing the Double Bass de Ludwig Streicher (15), 57 Studies in two volumes de Storch-Hrabe (15), Simplified Higuer Technique four double bass de Francesco Petracchi (8) o Nuovo Metodo de Isaia Billé (7).

La elección de qué elementos técnicos trabajar, depende en la mayoría de casos de la obra que se está trabajando en cada momento. Algunos de ellos se utilizan en prácticamente todas las clases, como el vibrato y las técnicas para la obtención del sonido (91’3\%), las escalas $\left(89^{\prime} 1 \%\right)$ o los golpes de arco (87\%). De forma más esporádica, se imparten otros elementos como la distribución de arco, articulación, digitación, etc. El tiempo dedicado en cada clase a la técnica del instrumento, es igualmente variable, dependiendo de la situación del alumno en cada momento, pero el profesorado coincide en que suele utilizar la mitad de la clase para estos temas.

Un $95^{\prime} 7 \%$ del profesorado destaca que es vital la correcta posición del instrumento con respecto al cuerpo. Esto coincide con la consciencia que se está formando en el mundo contrabajístico actual y que se ve en diferentes grupos de las redes sociales, sobre la importancia de fortalecer todos los músculos del cuerpo, con el objetivo de aumentar la resistencia y la flexibilidad a la hora de tocar el instrumento más grande de los instrumentos de cuerda.

A este respecto, un $65^{\circ} 9 \%$ del profesorado opina que es necesario realizar ejercicio para fortalecer el tono muscular y recomiendan a sus alumnos diversas actividades deportivas como la natación, yoga, cardio, Técnica Alexander, etc. Además, un 72’3\% realizan un calentamiento previo y estiramientos al finalizar cada clase que imparten 


\section{PAula Hernández Dionis}

En cuanto a la organización y dotación de material de los conservatorios con respecto a la enseñanza del contrabajo, se aprecia un claro descontento del profesorado. En muchos casos, debido a la falta de dotación económica necesaria por distintas causas y en otros debido a la falta de alumnos suficiente que justifiquen los gastos a realizar.

En lo referente a la dificultad para organizar cursos y masterclass, podemos observar diferentes opiniones en el siguiente gráfico:

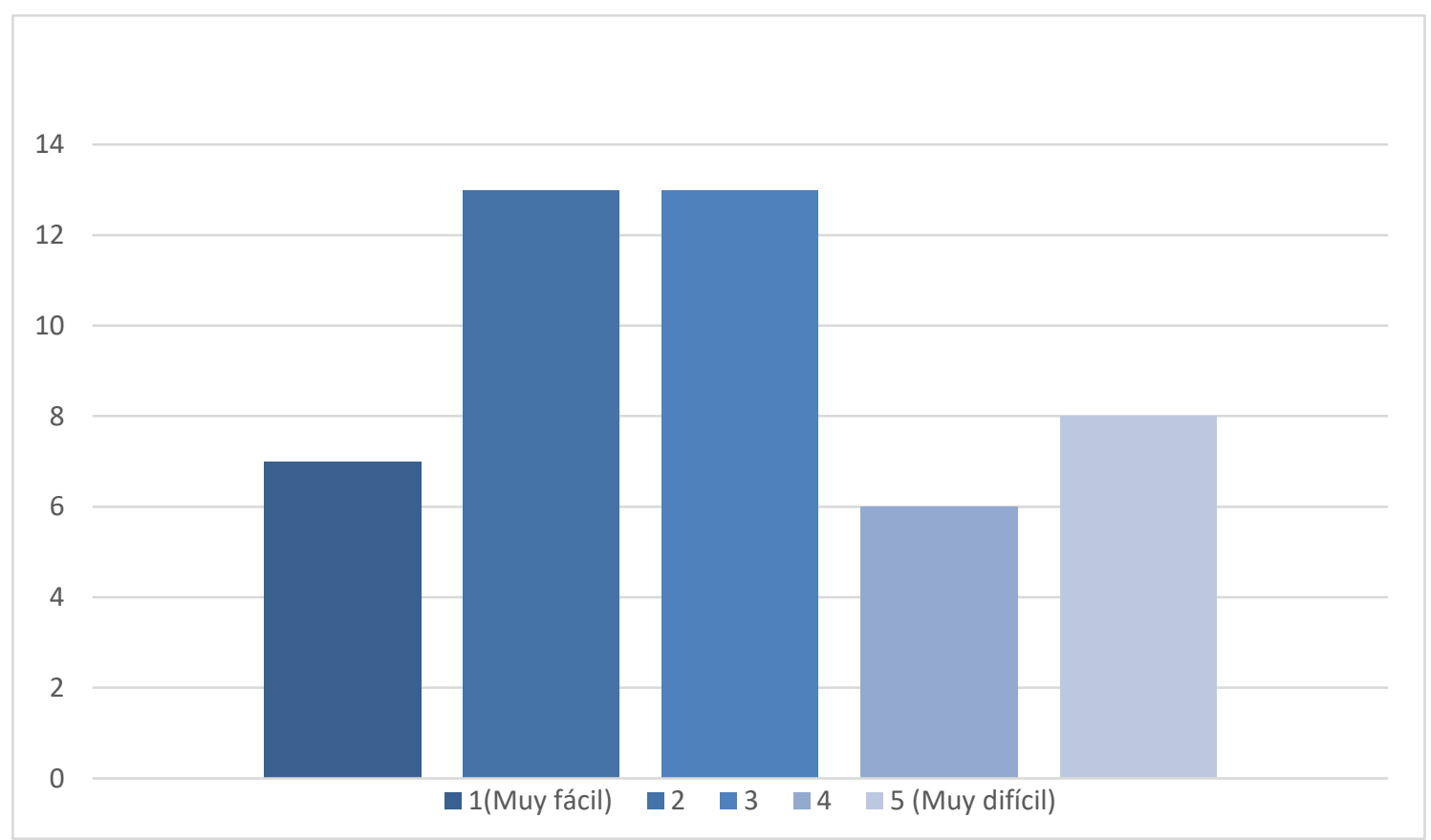

Fig. 1: Grado de posibilidad de organizar un curso o masterclass.

(Elaboración propia).

Entre los motivos que se esgrimen para opinar que es difícil o muy difícil la organización de este tipo de eventos, encontramos la dificultad que suponen los trámites administrativos, el poco alumnado matriculado en el centro, la situación socioeconómica familiar del alumno para sufragar de forma total o parcial los gastos, la falta de presupuesto y la disponibilidad de horario para realizarlo, tanto del profesorado como del alumnado.

Es por ello, que tan solo 17 profesores pudieron organizar un curso en el periodo escolar del curso 2018/2019. Además, nos encontramos con el asombroso dato de que 13 de ellos nunca han podido organizar ningún curso en sus años de docencia en los conservatorios. Entre los profesores que son más demandados para impartir estas masterclasses se incluyen: Xavier Sapiña, Ximo Clemente, Frano Kakarigi, Antonio García Araque, Hugo Valero o Antonio Torres.

En un $72 \% 3 \%$ de los conservatorios existen contrabajos de préstamo para sus alumnos, aunque en un $42^{\prime} 5 \%$ de ellos no hay suficientes para el número de alumnos que cursan estas enseñanzas. Estos instrumentos se prestan habitualmente a los alumnos matriculados en las 
EE, generalmente hasta que pueden adquirir uno propio o hasta que un alumno más pequeño lo necesita. En muchos casos, el alumnado puede practicar con los instrumentos del centro, sin salir del mismo, en horario no lectivo de la asignatura. Es de destacar que tan solo en un $46^{\circ} \%$ de los centros existen instrumentos de $1 / 8$, lo que dificulta enormemente los comienzos con el contrabajo. Solo en dos de estos centros tienen instrumentos de 1/16 y $1 / 10$ para sus alumnos.

Para poder impartir las clases correctamente, incluyendo las clases grupales o de orquesta, un $57^{\prime} 4 \%$ de los centros no tiene la cantidad de instrumentos necesarios. Además, un $34 \%$ de los mismos, no tiene la cantidad de banquetas suficientes para impartir docencia del instrumento y del resto de asignaturas donde se necesitan. Por ejemplo, cuando la actividad en la especialidad es intensa, coincidiendo en el tiempo las clases individual, colectiva y de orquesta, muchas veces no existen suficientes instrumentos y banquetas para todos ellos.

\section{CONCLUSIONES}

Los inicios, evolución y situación actual de la docencia del contrabajo en los Conservatorios Elementales y Profesionales en España, es un hecho que apenas ha sido investigado. Las referencias encontradas al respecto en fuentes primarias y secundarias son realmente escasas.

El hecho de ser el instrumento más grande de la familia de la cuerda, así como la poca cantidad de alumnado matriculado en los centros, hacen que la praxis de este instrumento sea más complicada que en otros instrumentos.

Sin embargo, se observa un gran auge en las enseñanzas de la especialidad de Contrabajo, con respecto a años anteriores y un crecimiento del interés palpable por el profesorado por mejorar la metodología que utiliza en sus centros, programando nuevas actividades, grupos y encuentros de contrabajo.

Asimismo, son varios los profesores que han diseñado nuevos métodos para el instrumento, como por ejemplo From the cello to the bass 1 y 2 (Hernández-Dionis, 2020a y b) o Contrabajo para niños (Valero, 2012), que actualmente desbancan a los métodos tradicionales que se venían utilizando hasta ahora. Sin embargo, se demanda por parte de los docentes, más material didáctico, especialmente para grupos de contrabajo de nivel inicial.

Todo esto, unido a la facilidad existente actualmente para conseguir instrumentos de pequeño tamaño $(1 / 16,1 / 10,1 / 8,1 / 4,1 / 2,3 / 4)$ que hace unos años apenas existían, hace que los alumnos de más corta edad puedan acceder a estudiar en los centros. A pesar de esto, la calidad de los instrumentos con los que cuentan las instituciones, ya sean para niños o adultos, es en la mayoría de ocasiones muy baja y en un estado de conservación deficiente, imposibilitando que el alumnado pueda sacar adelante elementos técnicos e interpretativos de cierta dificultad con la soltura necesaria y que la motivación del mismo decaiga. Lo mismo ocurre con la calidad de los arcos, ya sean en propiedad del alumnado o del centro. 


\section{Paula Hernández Dionis}

Se detecta en muchas ocasiones que, las aulas en las que se imparten clases de contrabajo, no son lo suficientemente grandes ni adecuadas, siendo todo esto un gran inconveniente tanto para el alumnado como para el profesorado.

Se podría considerar que la promoción del instrumento en los centros e instituciones es escasa, haciéndose un gran esfuerzo por el docente para captar al alumnado, incluso fuera de los límites de su conservatorio.

Por otro lado, aunque la calidad del profesorado de contrabajo es excelente, siendo la formación del mismo de nivel internacional, es palpable la necesidad de actualizar y formar al profesorado en las nuevas didácticas específicas que se salen de la rigidez establecidas en las programaciones académicas, tal y como muchos profesores solicitan.

\section{BIBLIOGRAFÍA}

Almenara, F. J. (2007). El contrabajo a través de la historia. Murcia: Ediciones Infides, S.L.

Ballesteros, M. (2004). El contrabajo. Una visión integral. Oviedo: Editorial Trabe.

Bisquerra, R. (1989). Métodos de investigación educativa. Guía práctica. Barcelona: Ediciones Ceac, S.A.

Conservatorio de Música "Cristóbal Halffter". (2020). Normativa. Historia de las enseñanzas artísticas de música en España Recuperado de: http://cpmhalffter.centros.educa.jcyl.es/sitio/index.cgi?wid_seccion=7\&wid_item $=169$

Crisanto, X. (1996). Algunas cuestiones relativas a la historia del contrabajo. Quodlibet: revista de especialización musical, 5, pp. 19-25. Recuperado de: https://ebuah.uah.es/dspace/bitstream/handle/10017/22236/algunas_g\%c3\%a1n dara_QB_1996_N5.pdf?sequence=1\&isAllowed $=y$

Crisanto, X. (2000). La escuela de contrabajo en España. Revista de Musicología, 23(1), pp. 147 186. https://doi.org/10.2307/20797637

Hernández-Dionis, P. (2017). Del violonchelo al contrabajo (Trabajo Final de Grado). Tenerife: Conservatorio Superior de Música de Canarias.

Hernández-Dionis, P. (2020). From the cello to the bass, vol. 1. Sevilla: Editorial Punto Rojo. Hernández-Dionis, P. (2020). From the cello to the bass, vol. 2. Sevilla: Editorial Punto Rojo. 
Streicher, L. (1997). My way of playing the double bass. Viena: Doblinger.

Robledo, L. (2001). La creación del conservatorio de Madrid. Revista de Musicología, 24(1/2), pp. 189-238. https:/doi.org/10.2307/20797694

Rodríguez, G., Gil, J. \& García, E. (1996). Metodología de la investigación cualitativa. Archidona: Ediciones Aljibe, S.L.

Ruiz, F. (2017). Manuel Verdeguer Beltrán. Descubrimiento de una Leyenda del Contrabajo. Valencia: Editorial Piles.

Valero, H. (2012). Contrabajo para niños. Canciones y fragmentos clásicos adaptados a contrabajo. Primera parte. Valencia: Piles Editorial de Música, S.A.

Fecha de recepción: 04/09/2020

Fecha de aceptación: 01/03/2021 